\title{
EARLY MIMD EXPERIENCE WITH A PLASMA PHYSICS SIMULATION PROGRAM ON THE CRAY X-MP
}

Clifford E. Rhoades, Jr.

MS Date

September 27, 1983

Printed February, 1986

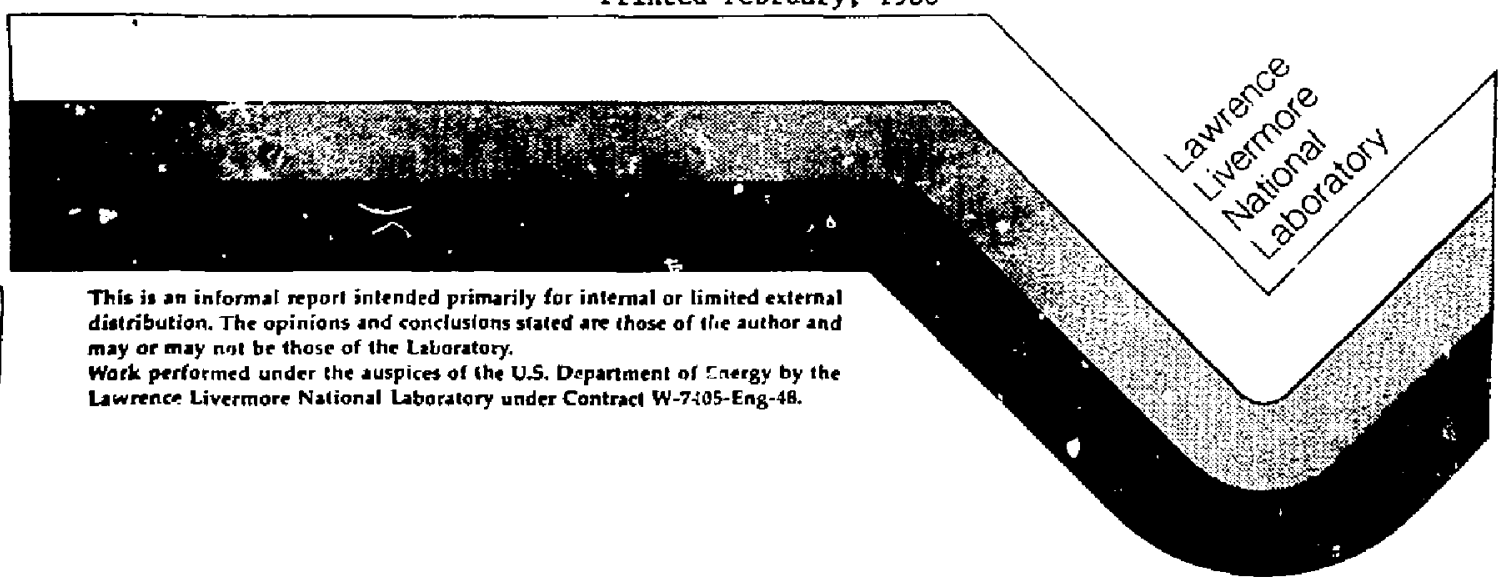




\title{
EARLY MIMD EXPERIENCE WTTH A PLASMA PHYSICS \\ SIMULATION PROGRAM ON THE CRAY X-MP*
}

\author{
Cufford E. Rhoades, Jr. \\ Theoreticel Physics Dlvision \\ Lawrence Livermore National Lihoratory \\ Livermore, CA 94550
}

September 27, 1983

\begin{abstract}
This paper describes some early experience with converting a plasma physics simulation program to the CRAY X-MP. a current multiple instruction, multiple data (MIMD) computer consisting of two prccessors with architecture similar to that of the CRAY-1. The computer program used in this study is an all Fortran version of SELF, a two species, one space, two velocity, electromagnetic, Newtonian, particle in cell, plasma simulation code. The approach to converting SELF to use both processors of the CRAY $X-M P$ is described in some detail. The resulting multiprocessor version of SELF is nearly a factor of two faster in real time than the single processor version. The multiprocessnr version obtains $58.2 \pm .1$ seconds of central processor time in $30 \pm .5$ seconds of real time. For comparison, the CRAY-1 execution time is 74.5 seconds. For SELF, which is mostly scalar coding, the CRAY X-MP is thout 2.5 times faster overall than the CRAY-1.
\end{abstract}

\section{DISCLAIMER}

\begin{abstract}
This repor was prepared as an acouunt of work sponsored by an agency of the United States Government. Neither the United States Government nor any agency thereof, nar any of their employes, makes any warranty, express or implied, or assumes any legal liability or responsibility for the rocuracy, completeness, of usefulness of any itformation, apparatus, product, or process disclosed. or represents that its use would not infringe privately owned rights. Reference herein to any specific commercial product. process, or service by trade mame, trademark. masulactuser, or otherwise does not necessarily constitute or imply its endorsement, recommendation, or favoring by the Unitod States Government or any agency thereol. The views and opinions of authors expressed hertin da not necessarily state or rellect those of the Unitod States Government or any agency thereof.
\end{abstract}




\section{INTRODUCTION}

The purpose of this paper is to document some of our earliest experience with running a physics computer program on a commercially available mutiple instruction. multiple data (MIMD) computer, the CRAY X-MP. ${ }^{2}$ We hope chereby to encourage others to explore the new world of parallel execution of physics codes. We begin by discussing our reasons for performing this study and for making our particular choice of physics program. We then establish our terminology and describe the plasma physles simulation code SELF. Cu* approach to parallelizing SELF and our results are dis sussed. Finally, our conclusions and recommendations for future work are presented.

\section{BACKGROUND}

Accessible, usable, ?".d useful MIMD computers are still very rare. In our view, to be useful, an MIMD computer must offer performance superior to that of the seven year old CRAY-1. ${ }^{2}$ Tr be usable, sufficient software must be provilsd. To be accessible, the:e needs to be a falrly wide distribution of richinos.

CRA. X-MPz are just now entering the field. Currently, Cray Research, Inc., nas one installad at Mendota. NASA Ames Research Center has just received delivery. Her Britanic Majesty's Atomic Weapens Research Establishment and the Los Alamos National Laboratory will each install a macilne du' 8 the fourth calendar quarter of 1983 . Other laboratories including the Laurence Livermure National Laburatory will follow in 1984. Iable 1 contains a list of the location of the first twtive mactines. Consequently. CRAY X-MP's are becumiag fai-ly widely distributed.

The usability issue is not so easily dismissed. Btcause of the heretofore dearth of MIMD machines, computational physicists, numeri ial analysts, and comouter programmers have little experience with runring real physics programs on such machines. Ivot only is a new set of performance related issues present, but there are new issues of logical correctness. language constructs, and operating concefts. We have so little experience that $w \in$ are in much the same position today with respect to the parallel world as we were in the late 1960 's with respect to the vector world.

This paper alse offers !ight on the subject of usefulness of the CRAY X-MP. The amount of work reouired of the computaticnsl physiclst to obtain a $25 \%$ speed up is zero. Tc obtain a factor of 2.5 speed up requires more work. For a broad cless of Monte Carlo codes, of which the particle is cell plasma sinulation is a leading example, the amount of work is minimal. 
TABLE 1. Curtent sites of CRAY X-MP machines.

\begin{tabular}{ll}
\hline Location & Number of machirs \\
\hline NASA - Ames & 1 \\
ECMWF & 1 \\
Chevron & 1 \\
Digital Production & 1 \\
Los Alamor National Laboratory & 2 \\
Department of Defense & $2+$ \\
CEZE, France & 1 \\
AWRE. Urited Kingdom & 1 \\
Germany & 1 \\
Lawrence Livermore National Laboratory & 1 \\
\end{tabular}

The computer program SELF used in this study is a two species, one space, two velocity, self consistent, electromagnetic, Newtonian, particle in cell. plasma simulation code. It was chosen for a number of reasons. While small in size, SELF is a real physics research tool in that a number of papers "4 have been published using it. The Fortran version of SELF used here contains the same physics as that used in published studies. It is a real code not a pale imitation or stripped down version of the real thing. SELF is extrumely well documented ${ }^{5}$ and was also used as a benchmark in a computcr acquisition. ${ }^{6}$

SELF is a member of a whole family of plasma simulation codes ${ }^{5,}$ ' and a direct progenitor of a very sophisticated multi-dimensional plasma simulation code called CCUBE. ${ }^{*}$ It most ways, SELF is typical of a whole class of plasma simulation codes, so that what we learn from the exercise of putting SELF on the CRAY X-MP can be directly applied to other codes. Finally, the author was familiar with SELF, had a copy on his shelf, and thought that it would be straightforward to run on the CRAY X-MP. In this, he was not disappointed.

\section{MUIJTITASKING}

Since the tenninology used to discuss parallel processing has not yet been standardized, this section establishes some of the common concepts and ternis. In general, we have attempted to iollow that suggested in the Cray Research, inc., Multitasking User's Guide." 
Multitasking is a mode of operation that provides for parallel processing of a single program by two or more processors. Parallelism may be achieved by dividing a program into two or raore parts that can be executed at the same time. A program that can efficiently be multitasked will see its wall clock time as measured from start to finish on a dedicated system, reduced when compared with an lóentical single task program.

It is commonly believed that the best theoretical gain that can be achieved from multitasking is a factor of $\mathbf{n}$ where $n$ is the number of processors. On real processors, this hypothesis is known to be untrue. The best theoretical gain may actually be greater than $n$ because of reduced memory conflicts, and inproved instruction buffer management.

In generai, less than a factor of $n$ improvement will be seen in real programs. A program may not be separable into parts which execute in the same amount of time. Even where identical code without explicit logical data dependencies executes with different input data, execution times will not be identical because of memory conflicts. Those parts that can be multitasked may have dependencies on one anothar that result in one or more tasks heving to wait until another completes some operation. Finally, depending on the primitives concepts provided by the compiler, library and operating system and on their efficiency of implementation, some amount of multitasking oyerhead will occur.

As indicated previously, the parallel worid is potentially a much more perilous world. Many of us have had programs go astray when only one compute task was : ing multitasked with one or more input/output operations. Asynchronous errors are especially difficult to track down, because sometimes you see thim, sometimes you don't - all depending on systern activity and task timing. When two or more parts of a program may be executed at the same time, the potential for even more timing problems arise. Again these problems are often not reproducibie. In addition, there are few tools available to help track them down. Worse. it is not entirely clear what tools would prove useful and usable.

Multitasking is today at the same state as vectorizing was in the late 1960 's. There is little automatic help in code analysis. There are many who belleve such analysis is impossible. And there are very few working at solving the problem. As with vectorization, so too with multitasking. Once adequate experlence is gained, we will be able to find the right concepts and figure ou. what primitive operations are useful and what tools are needed and what language con tructs are viable. At that point, at least some of the impossibie will become possible. I'uis paper is one of the first steps alor road to a better understanding of the multitasking world.

At the present time, only COS. ${ }^{10}$ the CRAY Operating System from Cray Research. Inc., supports multitasking on the CRAY X-MP. CTSS, the LTSS/NLTSS dual system and 
NLTSS do not yet support multitasking, COS, CFT, ${ }^{3+}$ the CRAY Fortran Complier, and their support 1fbraries define a number of useful concepts, chief among them are TASKS, LOCKS, and EVENTS.

For simplicity, we use onty the ask concept. The task primitive operations which we use, are TSK3TART and TSKWAIT. TSKSTART initiates a task and TSKWAIT waits for the task to complete. Table Il provides additional information.

TABLE 11. Desc :iption of TSKSTART and TSKWAIT.

\section{TSKSTART initiates a task}

CALL TSKSTART (task-array, name, list)

task-array A two or three word task control array to be used for this task. Word 1 must be set to the length of the array, either 2 or 3 . Word 3 , if used, must also be set. On return, word 2 will be set to a unique task identifier that must not be changed by the program.

name External entry point at which task execution is ts begin. This name must be declared EXTERNAL in the program or subroutine making the r.all to TSKSTART.

list

The list of arguments to be passed to the new task when it is entered. This list may be of any length.

TSKWAIT waits for the task to complete execution

CALL TSKWAIT (task-arTay)

task-array Task control array as defined above.

SELF

This section describes the SELF computer program for the one-dimensional numerical simulation of collisionless piasmas. SELF solves the Vlassov equation in very limited regions of phase space by means of a simple Monte Cario technique, that of following trajectories of simulation particles as they move under the influence of self-consistent electromagne tic fields.

A time-centered differmce approximation of the Lorentz force law, formulated in Newtonian form, is used to advance the particle positions and velocities. Onily the $X$ position of each particle is carred in the calculation, while both $X$ and $Y$ velocity components are used. In the limited region of phase space described by the code. 
velocities and positions can vary continuousiy up to the computer round-off error. The particles move through a periodic grid of uniform spacing, a particle disappearing out one end of the grid reappearing at the other end. Charge and current densities are calculated at cell centers by inverse linear interpolation of partic:e positicas and velocities. From these, electromagnetic flelds are determined at the cell edges using Maxwell's eq.lations. The particles are then moved using the linearly interpolated flelds. This loop is continued for as long as necessary to obtain a solution to the problem of interest.

To facilltate comparison of calculated results with those of others and to tncreas; program runing speed, the relatitristic "external" scaling shown in Table JII is used (Relativistic Scaling Scheme). The unit of time is $1 / \omega_{p}$, where ' $p$ is the plasma frequency. The unit of velocity is $c$, the speed of light; and hence the unit of distance is $c / \omega_{p}$. The electric and ragnetic fields have units

$$
\sqrt{4 \pi n e^{m} e^{c^{2}}}
$$

where $\mathrm{n}_{\mathrm{e}}$ is the mumber density of electrons and $\mathrm{m}_{\mathrm{e}}$ is the electron mass. This choice for the filds allows easy display of field energies in keV/particle for direct comparison with electron and ton temperatures. This scheme is "external" in the sense that restits are printed out in these units. In additical to this external scaling. SELF has "internal" scalings which are used further to increase computational speed. These are discussed below.

The organization of SELF is along the feilowing lines. There is a main program in which all conctants are defined and scale factors computed and from which the subroutines doing the actual calculations are called. The structure of the callins sequence is divided into four parts. First, the particle positlons and velocities are lsaded, with the particle positions distributed uniformly and the velocities distributed in a shifted Gaussian. In addition, arrays are zeroed and any other necessary initial conditions are set. Second, flelds are calculated. Third, the electrons are moved, then the ions are moved. After a number of iterations of field soiving followed by pushing of electrons aud ions, the program prints out appropriate energy diagnostics.

Printed output begins with a summary of all the initial parameters. All of these are first given in cgs units and a few are then given in machine units. Next, the time step number, the $X$ electric field energy, the $Y$ electric fleld energy, the total electrid field energy, the magnetic fleld energy, the average particle thermal energles in boti. dtrections for the electrons and lons are given for each time step. 
TABLE II. External scaling in SELF.

Variable in usual units

Dimensionless variable multiplied by scale factor

\begin{tabular}{rll} 
TIME & $\mathrm{t}(\mathrm{sec})$ & $\tilde{\mathrm{t}} *\left(1 / \omega_{\mathrm{p}}\right)$ \\
PARTICLE VELOCITY & $\mathrm{v}(\mathrm{cm} / \mathrm{sec})$ & $\tilde{\mathrm{v}} * \mathrm{c}$ \\
PARTICLE POSITION & $\mathrm{X}(\mathrm{cm})$ & $\tilde{\mathrm{X}} *\left(\mathrm{c} / \omega_{\mathrm{p}}\right)$ \\
ELECTRIC FIELD & $\mathrm{E}$ (statvolts) & $\tilde{\mathrm{E}} * \sqrt{4 \pi \mathrm{m}_{\mathrm{e}} \mathrm{m}_{\mathrm{e}} \mathrm{c}^{2}}$ \\
MAGNETIC FIELD & $\mathrm{B}$ (gauss) & $\tilde{\mathrm{B}} * \sqrt{4 \pi \mathrm{m}_{\mathrm{e}} \mathrm{m}_{\mathrm{e}} \mathrm{c}^{2}}$ \\
\hline
\end{tabular}

$\mathrm{n}_{\mathrm{e}}=$ electron number density

$\mathrm{m}_{\mathrm{e}}=$ electron mass

$c=$ speed of light in vacuum

$\omega_{p}=\sqrt{4 \pi n_{e} e^{2 / m}}=$ plasma frequency

$\mathrm{e}=$ charge on the electron

\section{DIFFERENCING SCHEME}

The differencing scheme used to move the particles assumes that particle positions. the electric fields, and the magnetic field are known at integral time steps and that particle velocities are known at odd half-integral time steps. So that current may be calculated after the particles have moved half their distance, the particles are pushed twice in a single time step.

Thus we use

$$
\begin{aligned}
& x^{n+1 / 2}=x^{n}+v_{x}^{n+1 / 2 \frac{\Delta t}{2}} \\
& x^{n+1} \Rightarrow x^{n+1 / 2}+v_{x}^{n+1 / 2} \frac{\Delta t}{2} \\
& v_{x}^{n+1 / 2}=v_{x}^{n-1 / 2} \\
& v_{x}^{n+1 / 2}=v_{x}^{n-1 / 2}+\frac{\Delta t}{1+\left(\frac{\Delta t}{2}\right)^{2}\left(B_{z}^{n}\right)^{2}}\left[E_{x}^{n}+v_{y}^{n-1 / 2} B_{z}^{n}+\frac{\Delta t}{2}\left(B_{z}^{n} E^{n}-v_{x}^{n-1 / 2} B_{z}^{n 2}\right)\right]
\end{aligned}
$$




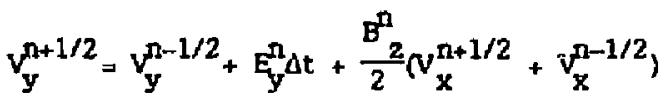

Equation (3) is first used to calculate $v_{x}^{n+1 / 2}$; then $v_{y}^{n+1 / 2}$ is calculated from Eq. (4). The partfcles are then pushed using Eq. (1) and the current in each cell calculated directly thereafter. The final push using Eq. (2) is then made; it is followed immediately by a calculation of the particle (charge) density in each cell. The area weighting scheme is used in computing charge density and current.

From the new particle charge densities, a new $E_{x}^{n+1}$ is determined by direct integration. The wave equation for $A_{y}$ in terms of $J_{y}$ is solved by

$$
\begin{aligned}
& A_{y}^{n+1 / 2}=2 A_{y}^{n+1 / 6}-A_{y}^{n-1 / 6}+\left(\nabla^{2} A_{y}^{n+1 / 6}+J_{y}^{n+1 / 6}\right)\left(\frac{\Delta t}{3}\right)^{2} \\
& A_{y}^{n+5 / 6}=2 A_{y}^{n+1 / 2}-A_{y}^{n+1 / 6}+\left(\nabla^{2} A_{y}^{n+1 / 2}+J_{y}^{n+1 / 2}\right)\left(\frac{\Delta t}{3}\right)^{2} \\
& A_{y}^{n+7 / 6}=2 A_{y}^{n+5 / 6}-A_{y}^{n+1 / 2}+\left(\nabla^{2} A_{y}^{n+S / 6}+J_{y}^{n+5 / 6}\right)\left(\frac{\Delta t}{3}\right)^{2}
\end{aligned}
$$

where we take

$$
\begin{aligned}
& \mathrm{J}_{y}^{n+1 / 6}=J_{y}^{n+1 / 2} \\
& J_{y}^{n+5 / 6}=J_{y}^{n+1 / 2}
\end{aligned}
$$

After $\mathrm{A}_{\mathrm{y}}$ is advanced using the above three equations, the magnetic field and the $\mathrm{Y}$ component of the electric fleld are given by

$$
\begin{aligned}
& B_{z}^{n+1}(X)=\left\{\left[A_{y}^{n+7 / 6}(X+1)+A_{y}^{n+5 / 6}(X+1)\right]-\left[A_{y}^{n+7 / 6}(X)+A_{y}^{n+5 / 6}(X)\right]\right\} / 2 \Delta X \\
& E_{y}^{n+1}(X)=\left\{\left[A_{y}^{n+5 / 6}(X+1)+A_{y}^{n+5 / 6}(X)\right]-\left[A_{y}^{n+7 / 6}(X+1)+A_{y}^{n+7 / 6}(X)\right]\right\} / 2(\Delta t / 3)
\end{aligned}
$$

\section{OPTIMIZATION}

To decrease running time, SELF has been optimized. In addition to the previously mentioned "external" scaling, the equations of motion have been rescaled.

The rescaling is accomplished as follows:

Redefine the following varlables: $\tilde{X}, \tilde{V}_{x}, \tilde{V}_{y}, \tilde{E}_{x^{\prime}} \tilde{E}_{y}$ and $\tilde{B}_{z}$ as shown below. 


$$
\begin{aligned}
& \tilde{x}=x / \Delta x \\
& \tilde{V}_{(x, y)}=V_{(x, y)} \frac{\Delta x}{2 \Delta x} \\
& \tilde{B}_{z}=-B_{z} \Lambda t \\
& \tilde{B}_{z} \text { (ion) }=B_{z} \Delta t \frac{m_{e}}{m_{1}}
\end{aligned}
$$

where $m_{e}=$ mass of electron and $m_{i}=$ mass of ion.

$$
\begin{aligned}
& \tilde{E}_{x}=-E_{x} \frac{(\Delta t)^{2}}{2 \Delta X} \\
& \tilde{E}_{y}=-\frac{E_{y}(\Delta t)^{2}}{2 \Delta X} \\
& \tilde{E}_{x} \text { (ions) }=-\frac{E_{x}(\Delta t)^{2}}{2 \Delta X} \frac{m_{e}}{m_{i}} \\
& \tilde{E}_{y} \text { (ions) }=E_{y} \frac{(\Delta t)^{2}}{2 \Delta X} \frac{m_{e}}{m_{i}}
\end{aligned}
$$

Then the particle equations of motion become

$$
\begin{aligned}
& \tilde{\mathrm{x}}^{\mathrm{n}+1 / 2}=\tilde{\mathrm{x}}^{\mathrm{n}}+\tilde{\mathrm{v}}_{\mathrm{x}}^{\mathrm{n}+1 / 2} \\
& \tilde{\mathrm{x}}^{\mathrm{n}+1}=\tilde{\mathrm{x}}^{\mathrm{n}+1 / 2}+\tilde{\mathrm{v}}_{\mathrm{x}}^{\mathrm{n}+1 / 2} \\
& \tilde{\mathrm{v}}_{\mathrm{x}}^{\mathrm{n}+1 / 2}=\tilde{\mathrm{v}}_{\mathrm{x}}^{\mathrm{n}-1 / 2}+\frac{1}{1+\left(\tilde{\mathrm{B}}_{\mathrm{x}}^{\mathrm{n}}\right)^{2}}\left[\tilde{\mathrm{E}}_{\mathrm{y}}^{\mathrm{n}}+\tilde{\mathrm{v}}_{\mathrm{y}}^{\mathrm{n}-1 / 2} \tilde{\mathrm{B}}_{z}^{\mathrm{n}}+\frac{\tilde{\mathrm{B}}_{z}^{n}}{2} \tilde{\mathrm{E}}_{\mathrm{y}}^{\mathrm{n}}-\tilde{\mathrm{v}}_{\mathrm{x}}^{\mathrm{n}-1 / 2} \frac{\tilde{\mathrm{B}}_{z}^{\mathrm{n}}}{2} \tilde{\mathrm{B}}_{z}^{\mathrm{n}}\right](22) \\
& \tilde{\mathrm{v}}_{\mathrm{y}}^{\mathrm{n}+1 / 2}=\tilde{\mathrm{v}}_{\mathrm{y}}^{\mathrm{n}-1 / 2}+\tilde{\mathrm{E}}_{\mathrm{y}}^{\mathrm{n}}-\frac{\tilde{\mathrm{B}}_{\mathrm{z}}^{\mathrm{n}}}{2} \tilde{\mathrm{v}}_{\mathrm{x}}^{\mathrm{n}+1 / 2}+\tilde{\mathrm{v}}_{\mathrm{x}}^{\mathrm{n}-1 / 3}
\end{aligned}
$$


Also, to save time in linear interpolation, the changes $\Delta \mathrm{E}_{\mathrm{x}^{\prime}} \Delta \mathrm{E}_{\mathrm{y}^{\prime}} \Delta \mathrm{B}_{\mathrm{z}}$ at each cell edge in the varlous flelds across th: cell in the direction of increasing $X$ from the edge are defined.

A brief description of subroutine functions is given in Table IV. Table $\mathrm{V}$ contains a simplified Warnier-Orr diagram of SELF.

TABLE IV. SELF Subroutine functions.

\begin{tabular}{|c|c|}
\hline Subroutine & Function \\
\hline \multirow[t]{3}{*}{ SELF } & Main program \\
\hline & Initial conditions \\
\hline & Calls other routines \\
\hline INPT & Prints out tnitial conditions \\
\hline INPTA & Prints out calculated parameters \\
\hline VEL9 & Sets initial particle distribution \\
\hline FIELD & Calculates $E_{x^{\prime}} E_{y^{\prime}} B_{z}$ from charge density and current \\
\hline \multirow[t]{2}{*}{ PUSH13 } & Particie pusher for electrons \\
\hline & Calculates charge density and current \\
\hline \multirow[t]{2}{*}{ PUSH14 } & Particle pusher for Ions \\
\hline & Calculates charge density and currant \\
\hline EPRINT & Prints out energies \\
\hline
\end{tabular}


TABLE V. Simplifted Warnier-OrT Dlagram of the ortglnal SELF

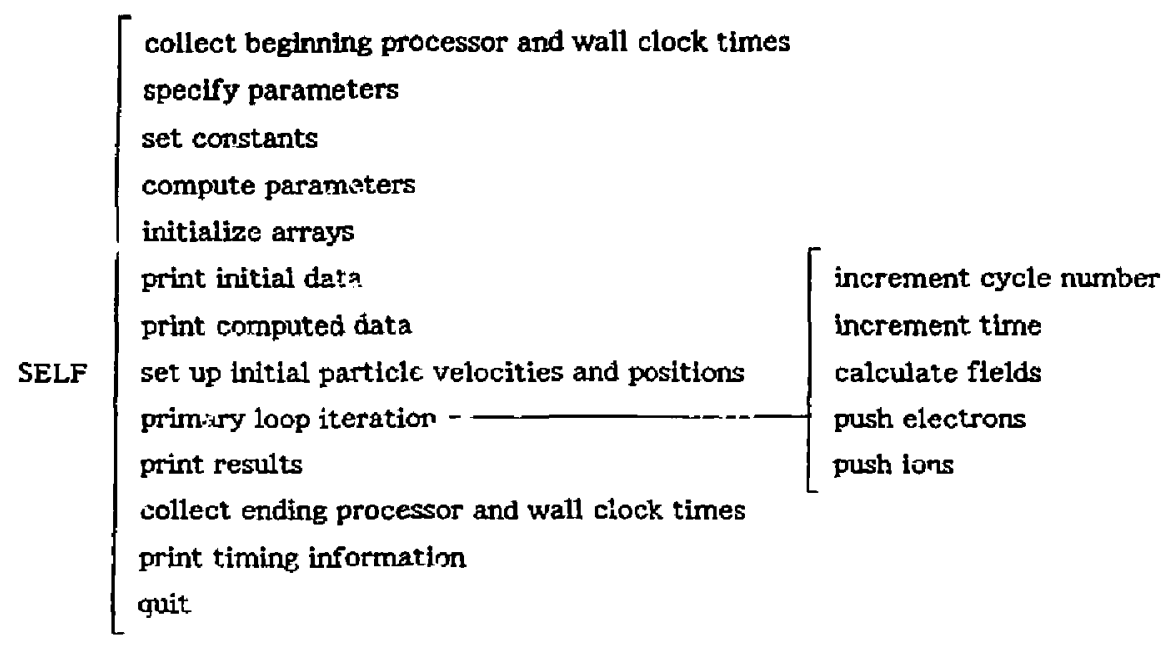

TABLE V. Simplified Warnier-Orr diagram of the two task SELF. Only the differences with respect to Table $V$. are shown.

SELF

primary loop iteration increment cycle mimber

increment tirs: 2

c. s: sulate fields

start task - push el_ctrons

push ions

wait for task to complete

compute total charge frrm

electron and ion charges

compute total current frc.n

electron and ion currents 
TABLE VII. FORTRAN of primary loop of original SELF.

130 ncy $=$ ncy+1

time $=\mathrm{dt}$ * ncy

call field (rho, Iy, exen, eyen, een, bzen)

call push13 (fho, jy, x1, vx1, vy1, venlx, veniy)

call push14 (tho, Iy, $x 2, v \times 2$, vy2, ven $2 x$, venzy)

if (ncy .lt. nt) go to 130

140 contimue

TABLE VII. Flow trace summary of original SELF for 50 timesteps.

\begin{tabular}{|c|c|c|c|c|c|c|}
\hline & Routine & Time & $\%$ & Called & \multicolumn{2}{|c|}{ Average $T$} \\
\hline 1 & SELF & 0.004464 & 0.11 & 1 & $\overline{0.004464}$ & \\
\hline 2 & WALCLK & 0.000208 & 0.01 & 2 & 0.000254 & Called by SELF \\
\hline-3 & CPCLK & 0.000052 & 0.00 & 2 & 0.009026 & Called by SELF \\
\hline 4 & TNPT & 0.007400 & 0.19 & 1 & 0.007400 & Called by SELF \\
\hline 5 & INPTA & 0.000887 & 0.02 & 1 & 0.000887 & Called by SELF \\
\hline 6 & VEL? & 0.235414 & 5.94 & 1 & 0.235414 & Called by SELF \\
\hline 7 & FIELD & 0.014776 & 0.37 & 50 & 0.000296 & Called by SELF \\
\hline 8 & PUSH13 & 1.815548 & 45.79 & 50 & 0.036311 & Called by SELF \\
\hline 9 & PUSH14 & 1.859253 & 46.89 & 50 & 0.371852 & Called by SELF \\
\hline 10 & EPRintT & 0.023150 & 0.58 & 1 & 0.023150 & Callee by SELF \\
\hline 11 & PRNTIM & 0.003390 & 0.09 & 1 & 0.003902 & Called by SELF \\
\hline$* * *$ & IOTAL & 3.964843 & & & & \\
\hline$=* *$ & Orerheas & 0.004781 & & & & \\
\hline
\end{tabular}

NOTE: These results were obtained on the CRAY 1-S 
TABLE LX. FORTRAN of primary loop of two task SELF.

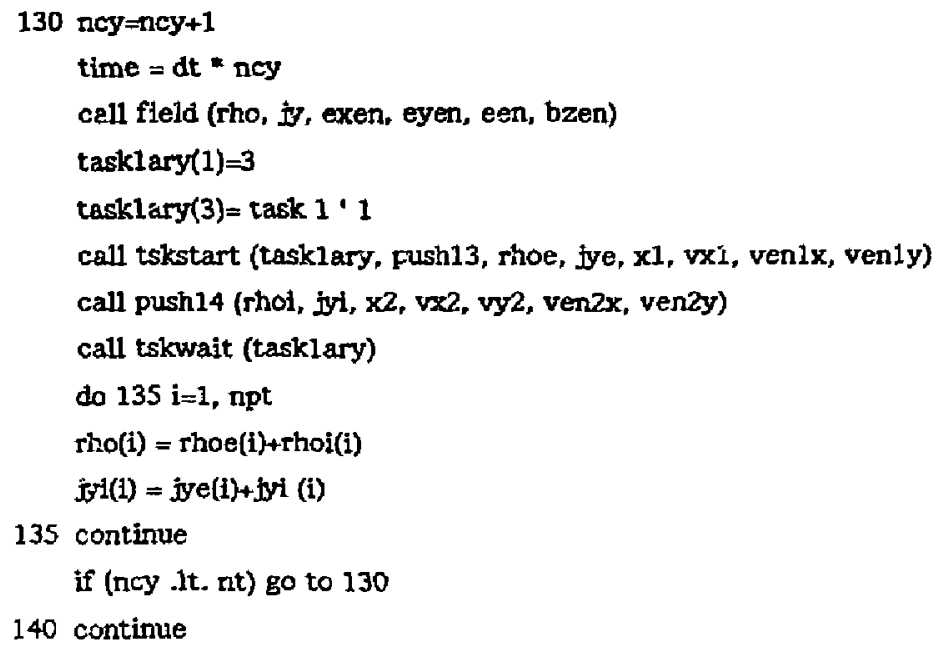

TABLE X. Timings for three runs of two task SELF.

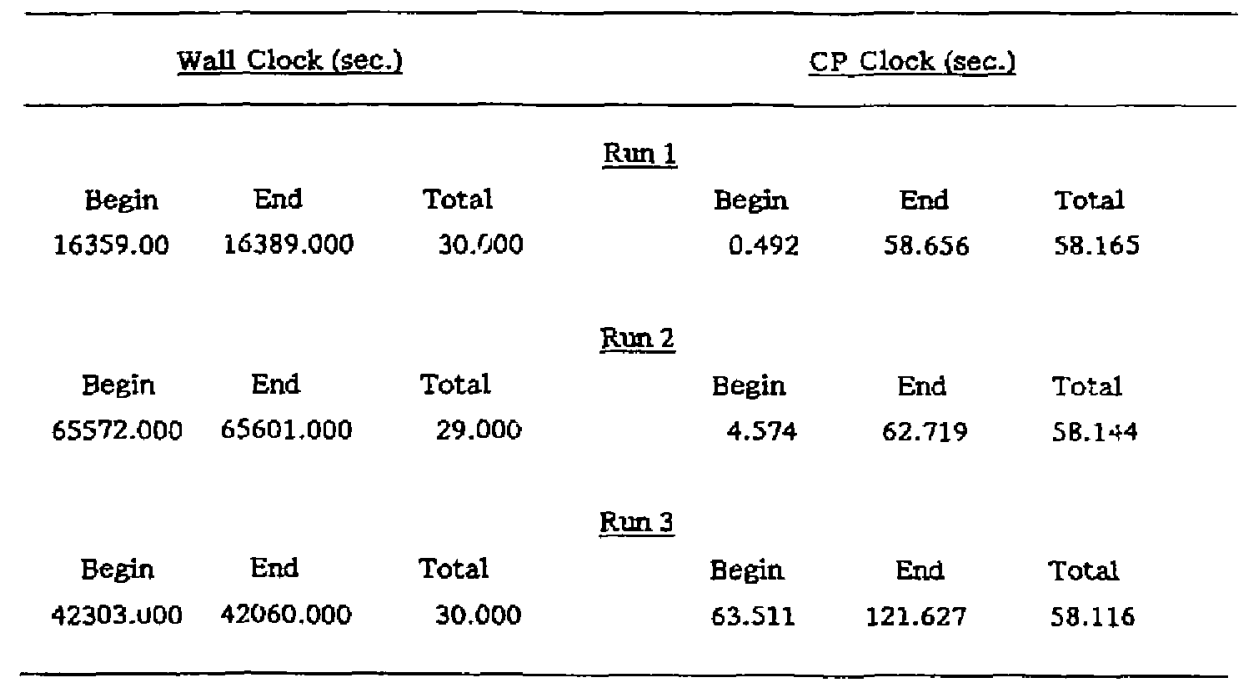


TABLE XI. 'Timings of single tasik SELF on CRAY I-S and CKAY X-MP.

\begin{tabular}{|c|c|c|c|c|c|}
\hline \multicolumn{3}{|c|}{ Wall Clock (sec.) } & \multicolumn{3}{|c|}{ CP Cloct (sec) } \\
\hline \multicolumn{6}{|c|}{ CRAY I-S } \\
\hline Begin & End & Total & Begin & End & Total \\
\hline 64635.000 & 66685.000 & 2050.000 & 0.651 & 75.167 & 74.517 \\
\hline \multicolumn{6}{|c|}{ CRAY $X-M P$} \\
\hline Begin & End & Total & Begin & End & Total \\
\hline 65550.000 & 65808.000 & 58.000 & 0.459 & 58.457 & 57.998 \\
\hline
\end{tabular}




\section{REFEKENCES}

1. CRAY X-MP Series Mainframe Reference Manual, Cray Research, Inc., Publication HR-0032 (19B2).

2. CRAY-1 Computer System Reference Manual, Cray Research, Inc., Publication 2240004 (1976).

3. K. A. Taggart, C. E. Rhoades, Jr., B. B. Godfrey, and H. C. Ives, "Second Order Effects in the Weibel Instability," Phys. Rev. Lett. 29, 1729 (1972).

4. C. E. Rhoades, Jr., B. B. Godfrey, and K. A. Taggart, "Weibel Instability, I: Solutions to Nonreiativistic s.eory and Numerical Simulation in One and a Half Dimensions," Proc. Int'1. Conf. on Waves and Instabilities in Plasmas, Innsbruck, Austria (1973).

5. B. B. Godirey, C. E. Hhoades, Jr., and h. A. Taggart, User's Manual AFWL One-Dinensional Plasma Simulation Particle Codes. Air Force Weapons Laboratory. Washington, D.C., AFWL-TR-72-47 (1972).

6. Air Force Weapons Laivoratory Computer Performance Specifications with Benchmarks, cornpleted November 1974, released in flrst draft August 1975 (unpublished).

7. C. E. Rhoades, Ir., and K. A. Taggarc, BOUNCE: User's Mamual, Air Force Weapons Laboratory, Washington, D.C., AFWL-TR-72-232 (1972).

8. M. M. Campbell. D. J. Sullivan, B. E. Godfrey, CCUBE User's Manual. AMRC-R-341, Mission Research Corporation, Albuquer que, NM (1982).

9. Wultitasking User's Guide, Cray Research, Inc., Publication (1983).

10. CRAY-OS (COS) Version 1 Reference Manual, Cray Research, Inc., Publication SR-0011 (1983).

11. FORTRAN (CFT) Reference Manual, Cray Research. Inc.. Publication SR-0009 (1983). 